\title{
Ascher's Syndrome - An Oral Medicine Perspective
}

\author{
Anuradha Ganesan, ${ }^{1, *}$, Gautham Kumar N $^{2}$ \\ ${ }^{1}$ Department of Oral Medicine \& Radiology, Madha Dental College \& Hospital, Chennai, India \\ ${ }^{2}$ Department of Periodontics, Madha Dental College \& Hospital, Chennai, India \\ *Corresponding author: anug77@yahoo.com
}

Received June 21; Revised June 27, 2014; Accepted July 02, 2014

\begin{abstract}
Aim: The aim of this case report is to highlight an extremely rare syndrome with oral manifestations from an oral medicine perspective. Background: Ascher's syndrome is an infrequent disease initially described by Ascher, an ophthalmologist, characterized by three more or less consistently associated abnormalities- double lip, blepharochalasis and enlargement of thyroid gland. This syndrome can be often missed out because of its rarity. Double lip is mostly seen in the upper lip and is also referred as macrocheilia. Blepharochalesis is localized angioedema of the eyelids. Thyroid enlargement is variable and not considered essential for the diagnosis of Ascher's syndrome. Double lip is of special interest to the dental profession as dentists may be the first clinician to encounter patients with this anomaly. Case description: An apparently healthy 14 year old female reported with the chief complaint of excessive tissue in relation to upper lip, which interfered with her mastication. Clinical examination revealed a cupid bow shaped soft tissue overgrowth in the mucosal part of the upper lip which compromised her esthetics and function. The finding of blepharochalesis associated with her right upper eyelid led to the diagnosis of Ascher's syndrome. Surgical correction of the excess lip tissue was done which considerably improved the appearance and function. Clinical significance: Detection of Ascher's syndrome can be missed out because of its rarity. Even though the patient may primarily have only an esthetic complaint when he/she presents with a double lip, the dentist may usually be at the forefront to diagnose this syndrome and initiate appropriate referrals and management.
\end{abstract}

Keywords: double lip, Ascher's syndrome, blepharochalesis, thyroid enlargement

Cite This Article: Anuradha Ganesan, and Gautham Kumar N, "Ascher's Syndrome - An Oral Medicine Perspective.” International Journal of Dental Sciences and Research, vol. 2, no. 4 (2014): 87-91. doi: 10.12691/ijdsr-2-4-4.

\section{Introduction}

Ascher's syndrome is an infrequent disease initially described by Ascher, an ophthalmologist from Prague in 1920.The disease is characterized by three more or less consistently associated abnormalities-double lip, blepharochalasis and enlargement of thyroid gland. [1] This syndrome can be often missed out because of its rarity. Around $50 \%$ of patients with Ascher's syndrome have double lip, as compared to only $10-50 \%$ showing thyroid enlargement $[2,3]$.

Double lip is a very rare oral anomaly and can be either acquired or congenital. [4] It does not have any age, sex or race predilection [5], however, a recent report suggests a male predilection of 7:1 [6], often in upper lip, but incidents of lower lip involvement are not uncommon. [7] Blepharochalasis is also a very rare eyelid disorder characterized by exacerbation and remission of eyelid edema resulting in atrophy of eyelid tissue, resulting in redundant folds over the lid margin. It affects only the upper eyelid and can be unilateral or bilateral [8].

Surgical correction of the lesion is often deemed necessary in spite of the symptomless nature of the syndrome because of the psychological factors associated with smile disfigurement. [9] This syndrome is of particular interest in dentistry as the dental professionals can diagnose without any unnecessary tests leading to diagnostic delays and treatment planning [7].

\section{Case Description}

An apparently healthy 14 year old female patient had visited our dental outpatient department with the chief complaint of thickened upper lip and also wanted cleaning of teeth. (Figure 1) History revealed that the thickening in the upper lip has been present from childhood and was creating embarrassment when she smiled. Initially she did not have much functional problems, but now, other than the esthetic problems, she had difficulty in speech and chewing. She also complained of occasionally chewing the extra soft tissue mass by her upper and lower anterior teeth which also gave her some pain. She also had periodic swelling of both the upper eyelids, which was painless and each episode lasting for 2-3 days and is been present for the past one year. There was no relevant medical history and no enlargement of thyroid gland. She did not have any lip sucking habit. There was no family history of double lip and no previous history of trauma or surgery. 


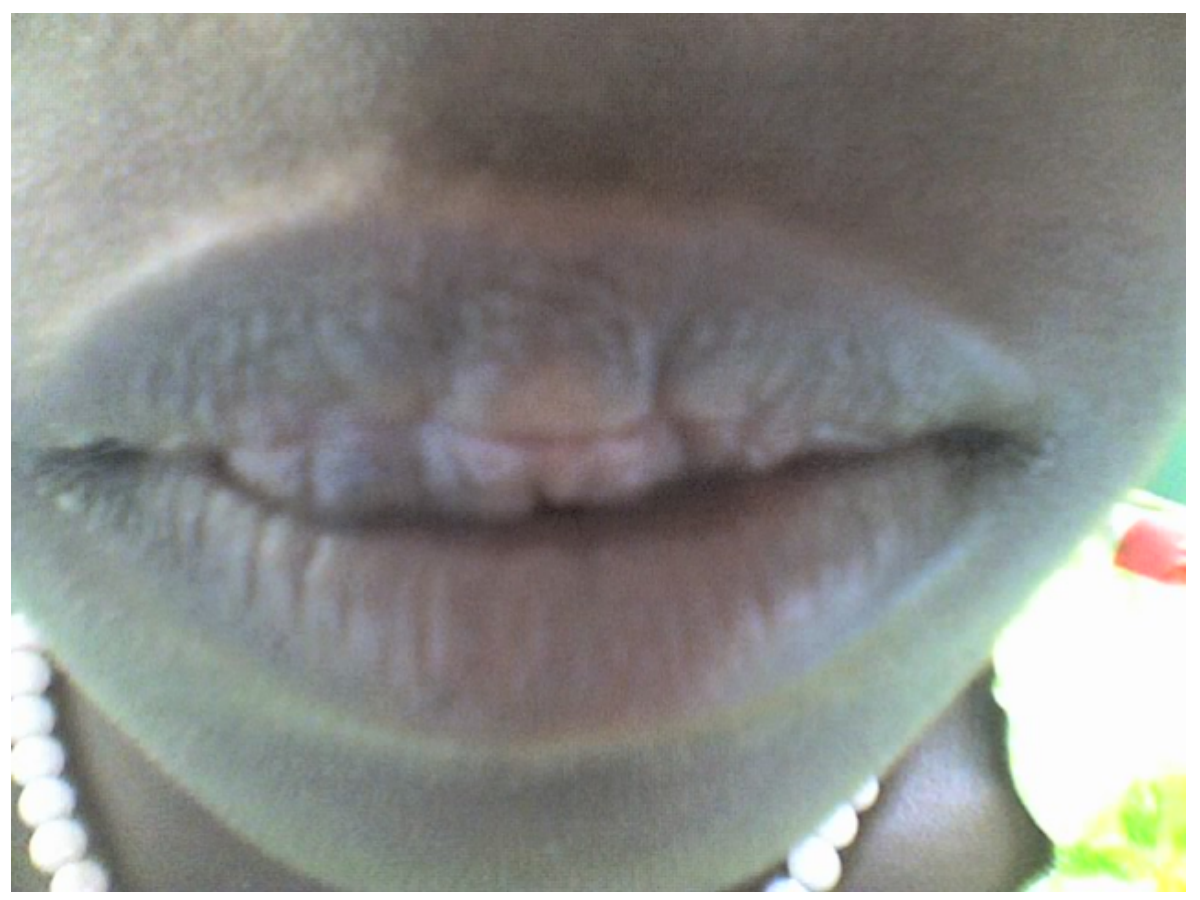

Figure 1. Extra oral close up photograph showing double upper lip

On clinical examination, when the patient smiled, there was bilateral extra fold of tissue with a midline constriction seen in the form of a cupids bow in the inner surface of upper lip. The buccal part of the double upper lip was measured as $3.9 \mathrm{~cm}$ by $1.3 \mathrm{~cm}$; the overlying mucosa was intact and smooth. (Figure 2) On palpation, it was soft and non compressible and also did not have any palpable masses or surface changes. There was no tenderness on palpation. The lower lip was normal. The excess tissue in the upper eyelid was more prominent laterally than medially and was present only on the right side, with the left eye appearing normal. (Figure 3) The lower eyelid and her eye movements were normal and so was her visual acuity, and there was no laxity of skin anywhere else in the body.

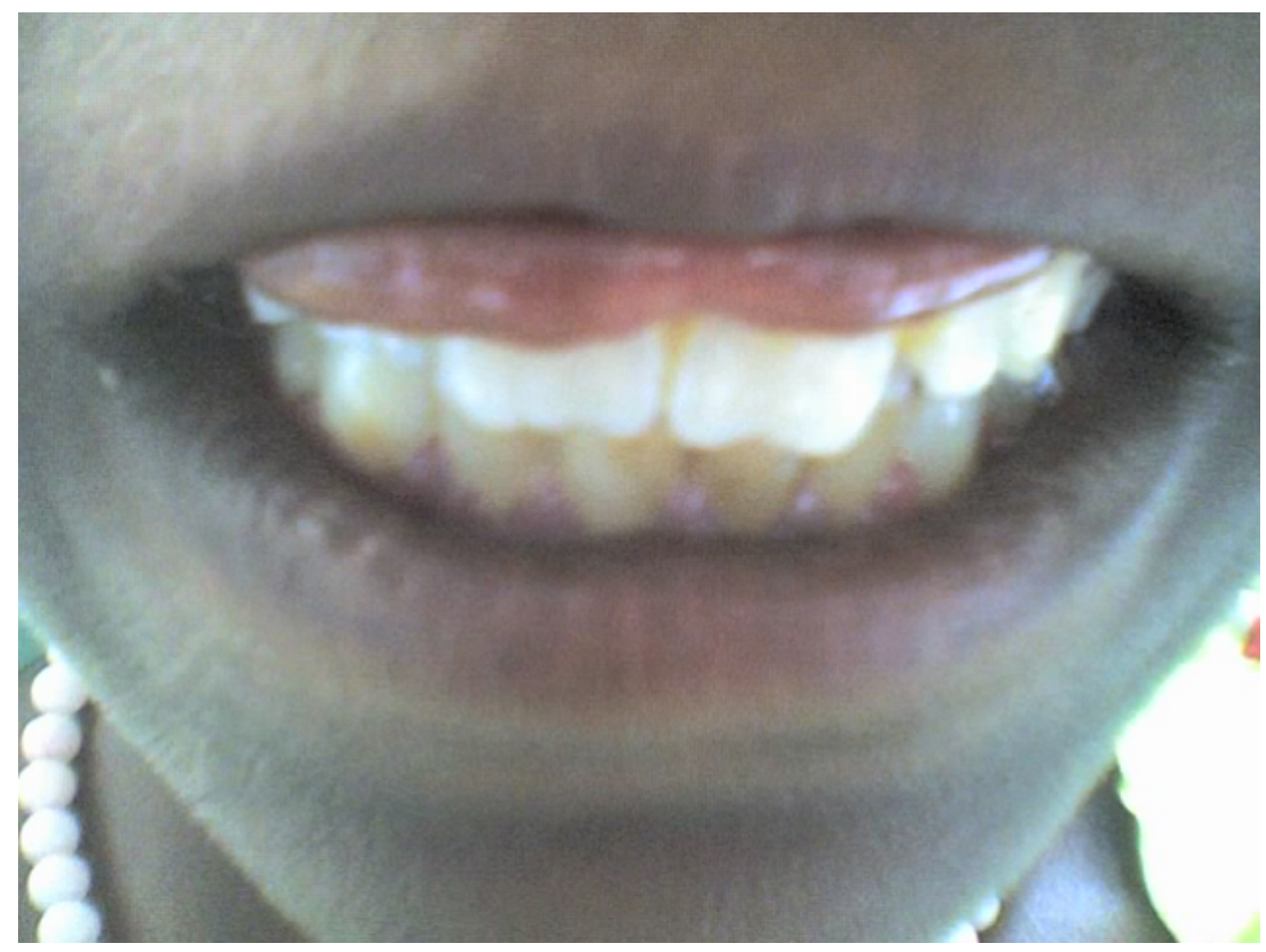

Figure 2. Cupid bow appearance of redundant upper lip 


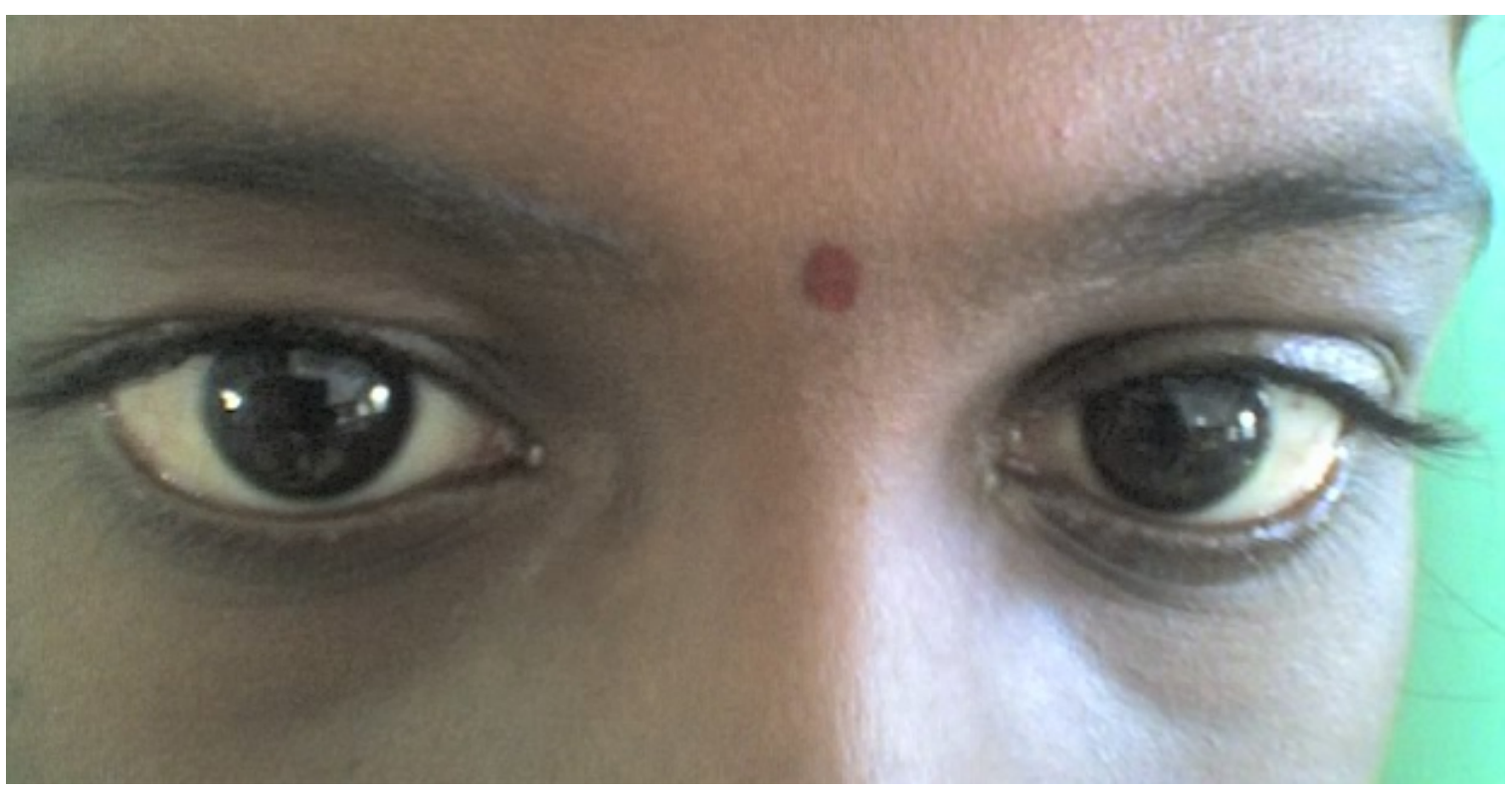

Figure 3. Blepharochalesis of upper right eyelid- notice the normal appearance of left eyelid

On dental examination patient had dental caries in 36, 46 and 26 and had extrinsic stains. Medical investigations were done and the patient did not have any systemic involvement. The blood, urine, ESR and thyroid function tests were done and were found to be normal. The differential diagnosis of double lip included all the form of enlargement of lip such as vascular tumours, hemangioma, lymphangioma, cheilitis glandularis, cheilitis granulomatosis, angioedema, inflammatory fibrous hyperplasia. $[6,10]$ The differential diagnosis of periorbital edema includes angioedema, blepharochalesis and cutix laxa. [11] With the above mentioned investigation, a final diagnosis of congenital double lip and blepharochalesis, a diagnosis of Ascher's syndrome was given. The thyroid enlargement associated with this syndrome is variable and may be present in rare cases only and is not essential in diagnosis of the syndrome [12].

The patient was referred initially to the department of periodontics for thorough oral prophylaxis to remove the extrinsic stains and then referred for the surgical removal of double lip. The surgical exision was suggested to the patient. Under local anesthesia, bilateral infraorbital nerve block was given. This prevents distortion of the tissue mass. The excess mass was removed by transverse elliptical incision. [13] The redundant mass was removed and also the minor salivary glands were removed to prevent future formation of mucocoele. The defect was closed in layers. Light compression dressing was applied for 24 hours after the procedure. No post operative problems were observed.

Histological examination revealed section of normal labial mucosa covered by stratified squamous epithelium. The underlying connective tissue exhibited numerous salivary glands with lymphocytic infiltration. Patient was recalled after one week and after two months. There was no recurrence and the cosmetic results were good (Figure 4). There was also no further increase in the size of thyroid gland.

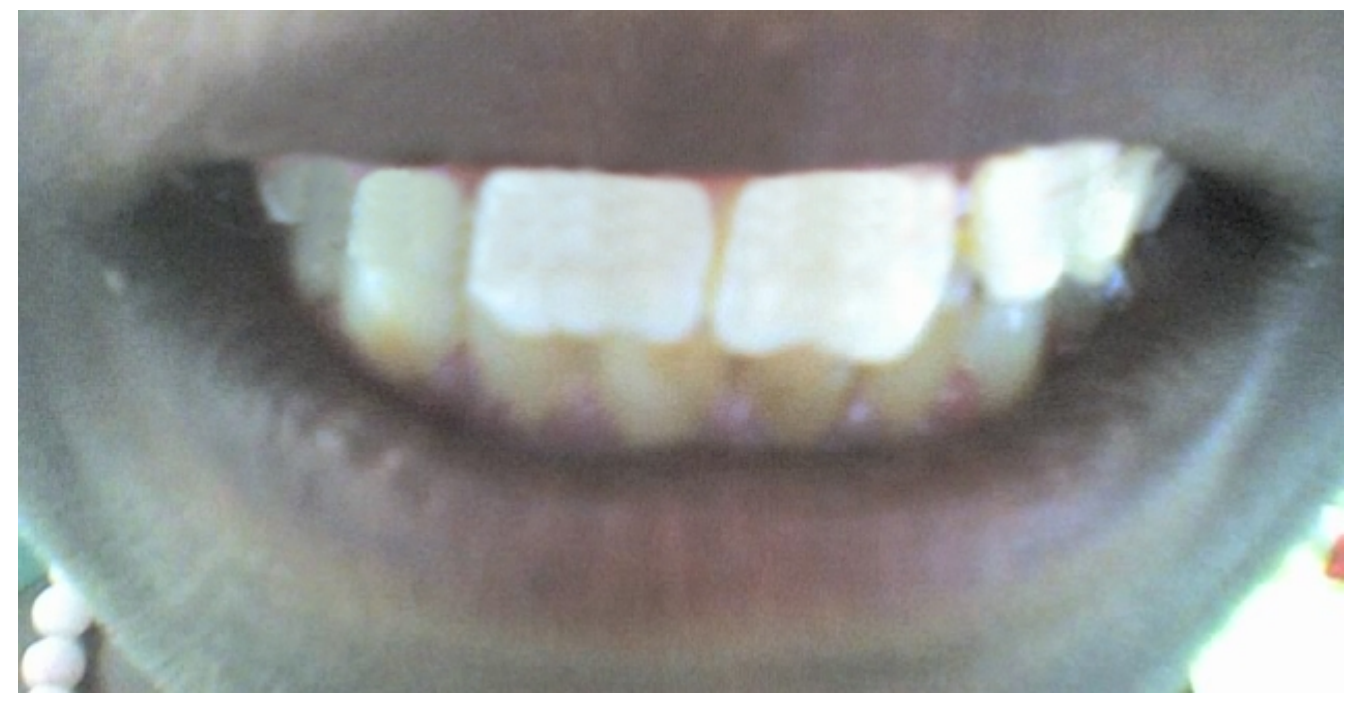

Figure 4. Post surgical view showing normal contours of lip

\section{Discussion}

Ascher's syndrome, the combination of double lip, blepharochalasis was first described by Laffer in 1909 [14], but in 1920, an ophthalmologist Ascher described 
the syndrome as a triad of double upper lip, blepharochalasis and non toxic thyroid enlargement. [1] The exact cause is unknown. Most cases are sporadic and rarely any family history has been recorded. It may be transmitted as an autosomal dominant disorder [15].

Blepharochalesis is present in more than $80 \%$ cases of this syndrome. It starts at puberty and usually both upper eyelids are involved. It is a form of localized angioedema with decreasing dermal elastin. There are 3 stages in blepharochalesis. First stage is edema stage with painless swelling of eyelid, second stage or atonic ptosis stage presents with ptosis due to dehiscence of levator aponeurosis, and third stage of ptosis adiposa presents with medial fat pad atrophy, orbital fat prolapse and lacrimal gland prolapse [16].

The recurrent episodes of eyelid swelling is usually non pitting and non erythematous edema. In most cases, frequency of the edema attacks in early stage in 3-4 a year, but weekly exacerbations have been reported. These episodes of eyelid swelling usually become less frequent as patient ages and eventually most cases enter a relatively quiescent stage. After several episodes, the eyelids become wrinkled, redundant, discoloured, thinned and laced by tortuous vessels. The wrinkled skin has a characteristic tissue paper appearance [17].

Double lip is a rare oral anomaly which is a fold of excess or redundant hypertrophic tissue of mucosal side of lip. It can involve both sides of upper lip bilateral or can be unilateral. It can affect lower lip also. It is also called macrochelia or hamartoma. [18] The congenital forms of double lip are believed to be due to persistence of sulcus between pars glabrosa and pars villosa of lip in the $2^{\text {nd }}$ or $3^{\text {rd }}$ month of gestation. During developmental stages, upper lip mucosa consists of 2 zones- outer zone, pars glaberosa, which is smooth and similar to skin and inner zone, pars villosa similar to oral mucosa. The central constriction seen is due to attachment of upper frenum. [18] The congenital double lip is noticed only after eruption of permanent teeth and is not evident when lip is at rest. But the excess tissue is seen beyond the vermilion border when the lip is tensed as in case of smiling or laughing resulting in characteristic cupid bow appearance. [15] Acquired double lip may be secondary to trauma or oral habits such as sucking the lip or between ill fitting dentures. [7] The patient with double lip as in our case can also develop speech problem and pronunciation of certain letters becomes difficult [1].

Thyroid enlargement is variable and may be present in $10-50 \%$ of cases of Ascher's syndrome and is not considered essential for diagnosis of syndrome. Also, thyroid involvement can occur years after eyelid involvement. In our case, the patient at present did not have any thyroid involvement and can be evaluated only if the patient comes for long term follow up. The present report describes a case of Ascher's syndrome with an incidence of double lip and blepharochalesis in a 14 year old female patient. Differential diagnosis of Ascher's syndrome includes vascular tumour, lymphangioma, angioedema, Miescher's syndrome, cheilitis granulomatosis, salivary gland tumours, inflammatory fibrous hyperplasia, sarcoidosis, and plasma cell cheilitis. Hemangioma, lymphangioma, angioedema, cheilitis glandularis and glandular granulomatosis do not have central demarcation in the lip. [10] Cheilitis glandularis is common in lower lip [3] and etiology is unknown, although familial inheritance and congenital predisposition bacterial infection and irritation from sun, chemicals and tobacco are other causes. [19] Cohen et al reported a case of concurrent double lip and cheilitis glandularis. [20] Association of double upper lip associated with hemangiomas and enlargement of thyroid gland has been described as CostaHaneman syndrome. [21] The double lip has also been reported with bifid uvula and cleft palate [9].

The treatment for blepharochalesis medical therapy includes anti histaminics, steroids, sympathomimetics, mast call stabilizers and acetazolamine with topical hydrocortisone and also oral doxycycline. Surgical procedures like lateral canthoplasty, exision or sculpting of prolapsed orbital fat. [11] The treatment of double lip involves surgical correction. Excision of redundant tissue can be made by elliptical incision, with plasty techniques, electrosurgical excision and triangular incision, mid moon incision. [22] The excised tissues on histopathology normally reveals stratified squamous epithelium with parakeratosis, minor salivary glands and moderate lymphocytic infiltration in underlying connective tissue with few muscle fibers [7].

\section{Conclusion}

Although Ascher's syndrome is a rare presentation, diagnosis of the syndrome through identification of the usual triad of clinical presentation would help guide the patient to the necessary specializations in medical field (ophthalmology and the ear nose and throat surgeon) for subsequent management. The finding of double lip, which could be a cause of esthetic embarrassment for the patient could bring the dentist to the forefront in terms of being the first to diagnose the condition.

\section{Clinical significance}

Detection of Ascher's syndrome can be missed out because of its rarity. Even though the patient may primarily have only an esthetic complaint when he/she presents with a double lip, the dentist may usually be at the forefront to diagnose this syndrome and initiate appropriate referrals and management.

\section{References}

[1] Pedo Paulo de Andrade Santos et al. Double lip surgical correction in Ascher's syndrome: Diagnosis and treatment of a rare condition. Clinics 2008; Oct 63 (5): 709-712.

[2] Beinhoffer, Piza katzer. Double lip in a patient with Ascher's syndrome. Eur J plast Surg 1998; 2: 310-313.

[3] Ali K. Ascher's syndrome- a case report and review of literature. Oral Surg Oral Med Oral Pathol 2007; 103: 26-28.

[4] Beena Roopak, Rohit. Congenital double lip - review of literature and case report. Ind J Clin Dent Jan 2012; 3 (1).

[5] Martins W D, Westphales F H, Sandrin R et al. Congenital maxillary double upper lip: review of literature and report of a case. J Can Dent Assoc 2004; 70: 466-468.

[6] Palma M C, Taub D I. Recurrent double lip: literature, review and report of case. Oral Surg Oral Med Oral Pathol Oral radiolog. Endod 107(2009), e 20-e 23.

[7] Usha Dabas, Vipin. K. Dabas. Clinical relevance,verification of systemic connection and management. Report of two cases of 
maxillary and mandibular double lip. Indian Journal of Dentistry 4(2013); 114-118.

[8] James, William, Berger et al. Andrews disease of skin. Clinical Dermatology (10 th edition) Saunders (2005); 515.

[9] Suliman M.T, Al Hassan M Double lip: Report of five cases and review of literature. Aesthetic surgery Journal 2007: 27: 289-91.

[10] Eski. M, Nisanci M, Aktas A et al. Congenital double lip: review of five cases. British journal and Maxillofacial Surgery 2007; 45: 68-70.

[11] Braakenburg A et al. Bilateral eyelid edema: cutis lassa or blepharochalasis. Ann Plast Surg 2000 Nov: 45(5): 538-40.

[12] Gomez-Duaso, Seane A J et al. Ascher's syndrome: report of 2 cases. J Oral Maxillo fac surg.1997; 55: 88-90.

[13] Kenny K F, Hreba J P, Dent C D. Bilateral redundant mucosal tissue of the upper lip. J Am Dent assoc 1990; 120(2): 193-4.

[14] Gorlin R J, Pindborg, Cohen J J et al. Syndromes of the head and neck. Mc Graw-Hill NewYork (2 ${ }^{\text {nd }}$ edition) 1976. pp 253-255.

[15] John Spencer M, Daniel, Congenital double upper lip: A case report and review of the literature. The Saudi Dental Journal (2010) 22, 101-106.
[16] Ramesh B A. Ascher's syndrome: review of literature and case report. Ind J Plastic Surg 2011; Jan-Apr 44 (1): 147-149.

[17] Daphna M K, Sara PM et al. The blepharochalasis syndrome. Survey of ophthalmology Mar2009; 54: 235-244.

[18] Sonia Goyal, Suhas Godhi, Sandeep Goyal: Non Syndromic Congenital Maxillary Double lip:a rare case. Journal oral health comm Dent 20082 (1): 10-12

[19] Dinesh Singh Chauhan, Yadavalli Guru Prasad. Congenital Maxillary Double Lip Chronicles of young scientists Vol3 Issue4 Oct-Dec 2012.

[20] Cohen D N, Green J G et al. Current anomalies: cheilitis glandularis and double lip. Oral Surg Oral Med Oral Path 1988; 66: 397-9.

[21] Costa Hanemann J A, Tostes- Oliveira D et al. Congenital double lip associated to hemangiomas: report of a case. Med Oral. 2004; 9: 155-158.

[22] Atzeni M, Cerevola E, Zaccheddu F et al. Surgical correction and $M R$ imaging of double lip in Ascher's syndrome: record of a case and review of literature. Eur Rev Med Pharmacol Sci2009; 13(4)309-311. 